\title{
聚乙烯吡咯烷酮存在时反相微乳液中水的状态 *
}

\author{
徐桂英 张 莉 毛宏志 鲍 猛 ${ }^{1}$ 卢 燕 1 \\ (山东大学胶体与界面化学教育部重点实验室,济南 250100; \\ ${ }^{1}$ 山东建材学院化学系, 济南 250022)
}

\begin{abstract}
摘要 运用傅立叶变换红外光谱 (FTIR) 研究不同分子量的聚乙烯吡咯烷酮 (PVP) 存在时, 十二烷基甜菜碱 $\left(\mathrm{C}_{12} \mathrm{BE}\right)$ /正庚烷 /正戊醇 / 水 ( I) 及二 (2-乙基) 已基磺化琥珀酸钠 ( $\mathrm{AOT}$ )/正庚烷 / 水 (II) 反相微乳液中水的 存在状态. 采用计算机分峰技术将微乳液中水分子的 $\mathrm{O}-\mathrm{H}$ 伸缩振动进行曲线拟合, ( I) 得到三个子峰, 分别位 于 $(3560 \pm 20) \mathrm{cm}^{-1},(3430 \pm 10) \mathrm{cm}^{-1},(3280 \pm 10) \mathrm{cm}^{-1}$ 附近; (II)得到四个子峰, 分别位于 $3618 \mathrm{~cm}^{-1}, 3550 \mathrm{~cm}^{-}$ ${ }^{1}, 3446 \mathrm{~cm}^{-1}$ 和 $3292 \mathrm{~cm}^{-1}$ 处. 尽管 PVP 均增溶于 $\mathrm{W} / \mathrm{O}$ 型微乳液中表面活性剂分子的极性基团附近, 却没有引 起长链间自由水的变化. 但由于两体系的差异, PVP 的存在, 导致微乳液 (I) 的本体水减少, 结合水增多, 却使体 系 (II) 的结合水减少, 本体水增多. 由于 $\mathrm{W} / \mathrm{O}$ 型微乳液中水与生物膜中水相似, 这些研究有助于理解生物膜界 面上的生物化学和生物物理现象。
\end{abstract}

关键词: 红外光谱, 聚乙烯吡咯烷酮 (PVP), 十二烷基甜菜碱 $\left(\mathrm{C}_{12} \mathrm{BE}\right)$, 二(2-乙基)已基磺化琥珀酸钠 $(\mathrm{AOT})$, 反相微乳液 $(\mathrm{W} / \mathrm{O})$

反相胶束或微乳液 $(\mathrm{W} / \mathrm{O})$ 是表面活性剂在非 极性溶剂中形成的聚集体。根据微乳液的拟相模 型, $\mathrm{W} / \mathrm{O}$ 型微乳液由油连续相、水核及界面膜三相 组成. 水核亦称为 水池” (water pool), 是加人的水 包在表面活性剂单层中形成的微水相. 水核可作 为微反应器进行多种反应 ${ }^{[1]}$, 而且, 这种水池类似于 酶中的极性囊, 可为底物的增溶及相互作用提供一 个独特的微环境. 水池中的水结构与生物膜中的 水及与蛋白质紧密结合的水的结构相似, 而与本体 水的性质相差很大 ${ }^{[2]}$. 因此, 这方面的研究引起了 许多化学家和生物学家的重视 ${ }^{[1,3-5]}$.

由于水溶性大分子与胶束和微乳液发生作用 而影响它们的微结构, 也就必然改变水的状态. 近 年来, 有关大分子存在下 $\mathrm{W} / \mathrm{O}$ 微乳液增溶水的状 态的研究成为倍受重视的课题 ${ }^{[5-9]}$. 但大分子对由 两性离子表面活性剂组成的微乳液结构的影响, 目 前尚未见报导。两性离子表面活性剂具有较高的 表面活性，低毒、低刺激，故而受到关注 ${ }^{[10,11]}$. 而 大分子/两性离子表面活性剂混合体系在食品、医
药、纺织及日用化学工业等领域有着广泛的应用前

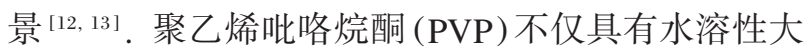
分子的一般性质, 而且毒性低, 生理相容性好, 在医 药、化妆品和食品工业中的应用越来越广泛 ${ }^{[14]}$.

FTIR 技术已成功地用于研究 $\mathrm{W} / \mathrm{O}$ 型微乳液 中水的结构和状态 ${ }^{[2,3]}$. 本文运用 FTIR 技术研究不 同分子量 PVP 存在时, 由两性离子表面活性剂十二 烷基甜菜碱 $\left(\mathrm{C}_{12} \mathrm{BE}\right) /$ 正庚烷 $\left(n-\mathrm{C}_{7} \mathrm{H}_{16}\right) /$ 正成醇 $\left(n-\mathrm{C}_{5} \mathrm{OH}\right)$ 和水组成的 $\mathrm{W} / \mathrm{O}$ 型微乳液增溶水的状 态变化, 并与水 $/ \mathrm{AOT} /$ 正庚烷 $\mathrm{W} / \mathrm{O}$ 微乳液作以 比较, 从而探讨了 PVP 与 $\mathrm{C}_{12} \mathrm{BE}$ 之间的相互作用 机理。

\section{1 实验部分}

\section{1 主要试剂}

十二烷基甜菜碱, $\mathrm{C}_{12} \mathrm{H}_{25} \mathrm{~N}^{+}\left(\mathrm{CH}_{3}\right)_{2}-\mathrm{CH}_{2}-\mathrm{CH}_{2}$ $\mathrm{CO}_{2}{ }^{-}\left(\mathrm{C}_{12} \mathrm{BE}\right)$, 太原发凯日用化工公司产品, 用前经 分析纯石油醚萃取, 并用丙酮重结晶两次, 测得其 等电点 $\mathrm{pI}=4.9$ 左右, 即当介质 $\mathrm{pH} \geqslant 4.9$ 时, $\mathrm{C}_{12} \mathrm{BE}$ 
以两性离子形式存在; 聚乙烯吡咯烷酮 (PVP: K15, $\mathrm{K} 30, \mathrm{~K} 90, \mathrm{~K} 120$ ) 均为美国特品公司产品, 用稀释型 粘度计测得前三者分子量分别为 8066、30300 和 518000; AOT (二 (2-乙基) 已基磺化琥珀酸钠) 为 Sigma 公司试剂, 纯度大于 $98 \%$; 正庚烷 $\left(n-\mathrm{C}_{7} \mathrm{H}_{16}\right)$ 和正戊醇 $\left(n-\mathrm{C}_{5} \mathrm{OH}\right)$ 均为国产分析纯试剂. 实验体 系均用三次蒸馏水配制。

\section{2 实验方法}

\section{2.1 微乳液配制}

首先绘制 $\mathrm{H}_{2} \mathrm{O} / \mathrm{C}_{12} \mathrm{BE} / n-\mathrm{C}_{5} \mathrm{OH} / n-\mathrm{C}_{7} \mathrm{H}_{16}[($ $\left.30.0 \pm 0.1)^{\circ} \mathrm{C}\right]$ 四组分拟三元相图, 找出 $\mathrm{W} / \mathrm{O}$ 型微 乳液区域 ${ }^{[15]}$. 然后选择含水量 $W_{0}=m_{\mathrm{H}_{2} \mathrm{O}} / m_{\mathrm{C}_{\mathrm{B}} \mathrm{BE}}=$ $15,\left(m_{\mathrm{H}_{2} \mathrm{O}}\right.$ 和 $m_{\mathrm{C}_{2} \mathrm{BE}}$ 分别为水和 $\mathrm{C}_{12} \mathrm{BE}$ 的摩尔数)、 $W_{\mathrm{C}_{\mathrm{i}} \mathrm{H}_{16}}=20 \%$ 及 $W_{\mathrm{C}_{12} \mathrm{BE}} / W_{\mathrm{C}_{\mathrm{S}} \mathrm{OH}}=3: 7\left(W_{\mathrm{C}_{12} \mathrm{BE}}\right.$ 和 $W_{\mathrm{C}_{\mathrm{s}} \mathrm{OH}}$ 分别 为 $\mathrm{C}_{12} \mathrm{BE}$ 和 $\mathrm{C}_{5} \mathrm{OH}$ 的质量) 的 $\mathrm{W} / \mathrm{O}$ 型微乳液体系, 考察含有和不含 PVP 时体系的变化.

将 AOT 溶于正庚烷中配成一定浓度的溶液, 取 一定量的该溶液放人数支试管中, 分别加人水或 PVP 水溶液,使水与 AOT 的摩尔比为 15 .

\section{2.2 红外光谱测定}

将含与不含 PVP 的微乳液分别盛人 $\mathrm{AgCl}$ 液池 中 (液池厚度选为 $0.025 \mathrm{~mm}$ ), 依次在 FTS-165 型傅 立叶变换红外光谱仪 (美国 BIO-RED 公司) 上测定 $900-4000 \mathrm{~cm}^{-1}$ 范围内的光谱图. 控制分辨率为 2 $\mathrm{cm}^{-1}$, 扫描 50 次. 用 Gaussion 曲线拟合程序对微乳 液中水分子的 $\mathrm{O}-\mathrm{H}$ 伸缩振动 $\left(3050-3800 \mathrm{~cm}^{-1}\right)$ 的红外光谱进行曲线拟合. 即用洛仑兹函数和高斯 函数的组合峰表征各个子峰, 将若干个子峰加起来 拟合成一个谱带, 将其与真实谱带相比较, 计算出真 实谱带与计算谱带间方差之和以表征二者的差别 . 调整各个子峰的位置、强度与峰形, 使这个方差之和 趋向最小, 从而把重叠峰分解成若干个子峰. 每个 子峰用四个参数来确定: 峰位 $v$ 、峰高 $h$ 、半峰宽度 $w$ 、高斯函数的百分数 $G$ (高斯函数与洛仑兹函数百 分数之和为 $100 \%$ ). 用这四个参数可以表征光谱中 的任何一个不重叠的吸收峰. 在确定每个子峰的相 对含量时, 以每个子峰的面积之和相加为 $100 \%$, 每 个子峰所占总面积的百分数即为其相对含量.

\section{2 结果与讨论}

\section{$2.1 \mathrm{PVP}$ 对 $\mathrm{H}_{2} \mathrm{O} / \mathrm{C}_{12} \mathrm{BE} / n-\mathrm{C}_{5} \mathrm{OH} / n-\mathrm{C}_{7} \mathrm{H}_{16}$ $\mathbf{W} / \mathbf{O}$ 微乳液 $(\mathrm{I})$ 中各种水含量的影响}

增溶于 $\mathrm{W} / \mathrm{O}$ 微乳液或反胶束水池中的水结构 及状态至今仍是一个有争议的问题. Zajac ${ }^{[10]}$ 用苂 光探针技术研究了由 AOT 所组成的 $\mathrm{W} / \mathrm{O}$ 微乳液, 提出微乳液体系中的水有两种存在形式: 一种是在 油水界面上表面活性剂分子或离子的结合水，另一 种是与体相水相似的本体水; Jain 等人 ${ }^{[3]}$ 采用红外 光谱技术研究的结果表明, 水分子的 $\mathrm{O}-\mathrm{H}$ 伸缩振 动的三个子峰分别对应于水的三种存在状态, 即除 结合水和本体水外, 还存在一种自由水, 这部分水以 单体或二聚体形式存在于表面活性剂长链之间, 与 周围无氢键作用; 而李泉等人 ${ }^{[16]}$ 则认为由 AOT 所 组成的 $\mathrm{W} / \mathrm{O}$ 微乳液水池中, 水分子至少有四种存 在状态: 本体水、自由水、阴离子结合水和阳离子结 合水. 四种状态对应于红外光谱中四个 $\mathrm{O}-\mathrm{H}$ 伸缩 振动峰.

我们对 $\mathrm{W} / \mathrm{O}$ 微乳液 $(\mathrm{I})$ 中水分子 $\mathrm{O}-\mathrm{H}$ 伸缩 振动的红外光谱测定表明, $\mathrm{O}-\mathrm{H}$ 伸缩振动峰可拟 合成三个子峰 (相关系数均为 0.9999 , 标准偏差为 $0.0011 \sim 0.0015)$, 分别位于 $(3560 \pm 20) \mathrm{cm}^{-1}$, $(3430 \pm 10) \mathrm{cm}^{-1},(3280 \pm 10) \mathrm{cm}^{-1}$ 附近 (见图 1). 处于表面活性剂长链之间的自由水, 其 $\mathrm{O}-\mathrm{H}$ 伸缩 振动峰位于 $3590 \mathrm{~cm}^{-1}$ 附近 ${ }^{[16]}$, 但考虑到微乳液滴 界面层中, 醇分子与表面活性剂分子的比例一般为 $2: 1^{[17]}$, 即醇分子大部分以二聚体形式存在. 这部 分醇分子的 $\mathrm{O}-\mathrm{H}$ 伸缩振动峰也位于 $3600-3500$ $\mathrm{cm}^{-1}$ 范围内 ${ }^{[18]}$. 可以认为, $(3560 \pm 20) \mathrm{cm}^{-1}$ 处的 拟合峰为戊醇分子和自由水共同作用所表现出的吸 收峰; $(3430 \pm 10) \mathrm{cm}^{-1}$ 处的峰则为存在于微乳液 水核中心部位的本体水所表现出的吸收峰, 此部分 水与纯水相似, 其分子间发生氢键缔合, 以多聚体的 形式存在; 而 $(3280 \pm 10) \mathrm{cm}^{-1}$ 处的吸收峰则可认为 是结合水的 $\mathrm{O}-\mathrm{H}$ 伸缩振动峰. 此结合水包括 $\mathrm{C}_{12} \mathrm{BE}$ 分子中 $-\mathrm{N}^{+}-$和 $-\mathrm{CO}_{2}^{-}$两种离子的结合水. 由于 $-\mathrm{N}^{+}-$周围有四个烷基, 空间障碍阻碍水分子 与这一大阳离子配位 ${ }^{[19]}$, 所以结合水很少. 水分子 与 $-\mathrm{N}^{+}$- 结合, 其 $\mathrm{O}-\mathrm{H}$ 原子间的电子云密度减 小, 红外吸收峰处于较低波数; 而与 $-\mathrm{CO}_{2}^{-}$作用的 水分子, 其 $\mathrm{O}-\mathrm{H}$ 原子间的电子云密度增大, 红外吸 

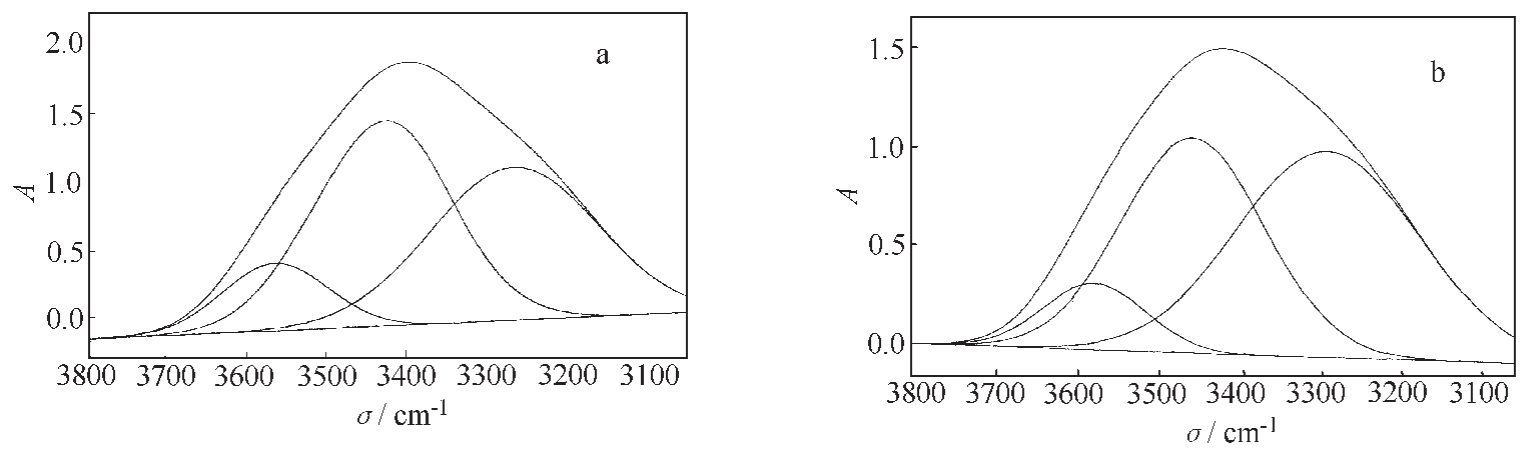

图 $1 \mathrm{H}_{2} \mathrm{O} / \mathrm{C}_{12} \mathrm{BE} / n-\mathrm{C}_{5} \mathrm{OH} / n-\mathrm{C}_{7} \mathrm{H}_{16}$ 微乳液中水分子的 $\mathrm{O}-\mathrm{H}$ 伸缩振动红外光谱曲线拟合结果

Fig. 1 Curve-fitted results of $\mathrm{O}-\mathrm{H}$ stretching band of $\mathrm{H}_{2} \mathrm{O}$ in microemulsion of $\mathrm{H}_{2} \mathrm{O} / \mathrm{C}_{12} \mathrm{BE} / n-\mathrm{C}_{5} \mathrm{OH} / n-\mathrm{C}_{7} \mathrm{H}_{16}$ a) Without PVP b) With $20.0 \mathrm{~g} \cdot \mathrm{L}^{-1} \mathrm{PVP}-\mathrm{K} 30$

收峰处于较高波数. 在此中性介质中, $\mathrm{C}_{12} \mathrm{BE}$ 分子 以内盐形式存在, 所以 $-\mathrm{CO}_{2}-$ 结合水的能力也较 弱. 另外, 由于部分醇分子与表面活性剂分子一起 形成界面膜, 醇羟基对水分子也有一定的结合能力 . 这些作用的综合结果, 使得结合水的红外吸收峰 位于 $(3280 \pm 10) \mathrm{cm}^{-1}$ 处.

按相同方法测得含有 $20.0 \mathrm{~g} \cdot \mathrm{L}^{-1} \mathrm{PVP}$ 时, 微 乳液的 $\mathrm{O}-\mathrm{H}$ 伸缩振动谱图. 结果表明, $\mathrm{PVP}$ 的加 人对所有红外吸收峰的位置和半峰宽均无明显影 响, 以 PVP- K30 体系为例 (图 1.b), PVP 的加人使 峰高降低, 且 PVP 分子量越大, 峰高降低幅度越大 （见表 1). 峰高降低, 即体系的红外吸收峰强度减 弱. 此变化趋势与体系中含水量减少对红外吸收 峰强度的影响类似. 因此, 可以认为 PVP 的加人, 使微乳液水核中的本体水含量减少. 由含不同分 子量 PVP 微乳液中 $\mathrm{O}-\mathrm{H}$ 伸缩振动峰, 求得各种水 的相对含量结果 (见表 2)进一步证明了这一点. 表 2 的数据表明, PVP 的加人对自由水的影响较小, 但 PVP 分子量越大, 微乳液水核中的本体水含量越 少, 结合水含量越多. 此结合水应是 $\mathrm{C}_{12} \mathrm{BE} 、 n-\mathrm{C}_{5} \mathrm{OH}$ 和 PVP 三种分子与水分子作用的综合结果. 因为

\section{表 1 含 $20.0 \mathrm{~g} \cdot \mathrm{L}^{-1}$ 不同 PVP 时水分子的 $\mathbf{O}-\mathrm{H}$ 键伸缩振动峰的高度}

Table 1 Effect of PVP on the peak high of curve fitted for $\mathrm{O}-\mathrm{H}$ stretching band of water

\begin{tabular}{cccccc}
\hline PVP & & K15 & K30 & K90 & K120 \\
\hline Peak a & 0.492 & 0.337 & 0.336 & 0.250 & 0.223 \\
Peak b & 1.485 & 1.263 & 1.092 & 0.899 & 0.786 \\
Peak c & 1.107 & 1.029 & 1.045 & 0.670 & 0.567 \\
\hline
\end{tabular}

PVP 为水溶性大分子, 其吡咯烷酮基与水分子有较 强的作用, 故可被增溶于水池中或液滴极性头周 围, 从而影响水分子的 $\mathrm{O}-\mathrm{H}$ 键伸缩振动的强度.

\subsection{PVP 对 $\mathrm{H}_{2} \mathrm{O} / \mathrm{AOT} / n-\mathrm{C}_{7} \mathrm{H}_{16} \mathrm{~W} / \mathrm{O}$ ( II) 微 乳液中各种水含量的影响}

AOT 在非极性溶剂中有着极有趣的性质和应 用, 而且不需助表面活性剂存在, 就可形成反相微 乳液. 因此, AOT W/O 微乳液的研究引起人们广 泛的兴趣 ${ }^{[1]}$. 利用计算机分峰程序对 AOT W/O 微 乳液中水分子的 $\mathrm{O}-\mathrm{H}$ 伸缩振动红外光谱进行曲 线拟合的结果表明, 无论存在 PVP 与否, 其 AOT $\mathrm{W} / \mathrm{O}$ 微乳液中的水分子 $\mathrm{O}-\mathrm{H}$ 伸缩振动均可分成 四个峰. 图 2 示出了含与不含 PVP 时, 微乳液中水 分子的 $\mathrm{O}-\mathrm{H}$ 振动峰拟合的结果. 四个子峰分别位 于 $3618 \mathrm{~cm}^{-1} 、 3550 \mathrm{~cm}^{-1} 、 3446 \mathrm{~cm}^{-1}$ 和 $3292 \mathrm{~cm}^{-1}$ 附近. 这四个 $\mathrm{O}-\mathrm{H}$ 伸缩振动峰与微乳液中水分子 的四种状态相对应. 根据李泉等人的观点 ${ }^{[16]}$, $\mathrm{H}_{2} \mathrm{O} / \mathrm{AOT} / n-\mathrm{C}_{7} \mathrm{H}_{16} \mathrm{~W} / \mathrm{O}$ 微乳液中水的四种存在 状态分别为: 表面活性剂长链间的自由水、磺酸根 的结合水、钠离子的结合水及水核中心部分的本体 水. 处于表面活性剂长链间的自由水, 由于它与表

表 2 含 $20.0 \mathrm{~g} \cdot \mathrm{L}^{-1}$ 不同 PVP 时微乳液中 各种水的相对含量

Table 2 Effect of PVP on the various water content in microemulsion

\begin{tabular}{cccccc}
\hline PVP & & K15 & K30 & K90 & K120 \\
\hline Bound water(\%) & 41.3 & 47.7 & 47.2 & 51.7 & 56.8 \\
Bulk Water(\%) & 46.8 & 42.2 & 41.6 & 35.1 & 33.2 \\
Free water(\%) & 11.9 & 10.1 & 11.2 & 13.2 & 10.0 \\
\hline
\end{tabular}



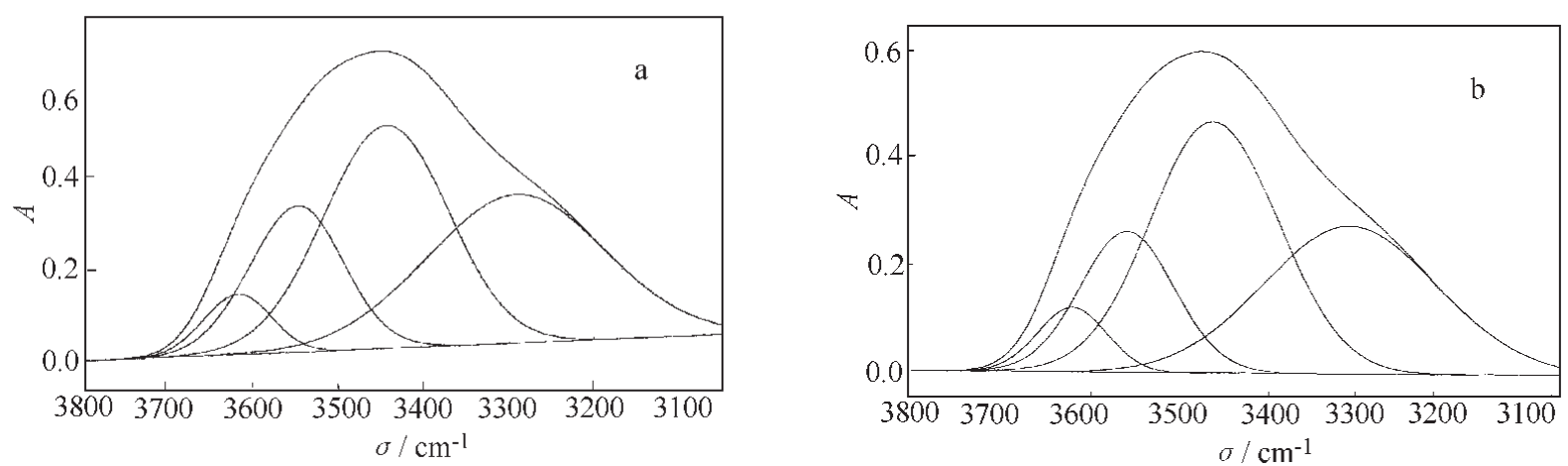

图 $2 \mathrm{H}_{2} \mathrm{O} / \mathrm{AOT} / n-\mathrm{C}_{7} \mathrm{H}_{16}$ 微乳液中水分子的 $\mathrm{O}-\mathrm{H}$ 伸缩振动红外光谱的曲线拟合结果

Fig. 2 Curve-fitted results of $\mathrm{O}-\mathrm{H}$ stretching band of $\mathrm{H}_{2} \mathrm{O}$ in microemulsion of $\mathrm{H}_{2} \mathrm{O} / \mathrm{AOT} / \boldsymbol{n}-\mathrm{C}_{7} \mathrm{H}_{16}$ a)Without PVP; b)With $20.0 \mathrm{~g} \cdot \mathrm{L}^{-1} \mathrm{PVP}-\mathrm{K} 30$

面活性剂分子及周围环境无相互作用，以单体或二 聚体形式存在, 其 $\mathrm{O}-\mathrm{H}$ 伸缩振动的吸收峰位于 $3617 \mathrm{~cm}^{-1}$ 处; $3550 \mathrm{~cm}^{-1}$ 处的拟合峰为磺酸根结 合水的 $\mathrm{O}-\mathrm{H}$ 振动峰; 存在于水核中心部分的本体 水与纯水相似, 其分子间有氢键作用 ${ }^{[20]}$, 这种水分 子的 $\mathrm{O}-\mathrm{H}$ 伸缩振动频率在 $3446 \mathrm{~cm}^{-1}$ 附近; 钠离 子结合水的 $\mathrm{O}-\mathrm{H}$ 伸缩振动峰位于 $3292 \mathrm{~cm}^{-1}$ 处.

含 $20.0 \mathrm{~g} \cdot \mathrm{L}^{-1}$ 不同分子量 PVP 时, 微乳液中 水分子的 $\mathrm{O}-\mathrm{H}$ 振动峰拟合曲线与不含 PVP 者相 似, 其相关系数均在 0.999 以上, 标准偏差为 0.00114 左右, 说明拟合曲线与实验曲线吻合得很 好. PVP 的加人未能明显改变各种状态水的 $\mathrm{O}-\mathrm{H}$ 伸缩振动峰的位置. 表 3 列出了含有 $20.0 \mathrm{~g} \cdot \mathrm{L}^{-1}$ 不同 PVP 时, AOT W/O 微乳液中 $\mathrm{O}-\mathrm{H}$ 振动的峰 位置. 可见, 在所研究的分子量范围内, PVP 的加 人均未明显影响各种状态水的 $\mathrm{O}-\mathrm{H}$ 伸缩振动峰的 位置. 但伸缩振动峰的高度随 PVP 分子量增大而 降低, 例如, 不含 PVP 时, AOT W/O 微乳液中水 分子的 $\mathrm{O}-\mathrm{H}$ 振动总峰的高度为 0.72 , 加人 20.0

\section{表 3 含不同分子量 PVP 时 $\mathrm{H}_{2} \mathrm{O} / \mathrm{AOT} / n-\mathrm{C}_{7} \mathrm{H}_{16}$ $\mathbf{W} / \mathbf{O}$ 微乳液中 $\mathbf{O}-\mathbf{H}$ 伸缩振动峰的位置}

Table 3 Effect of PVP on the position of curve-fitted

\begin{tabular}{cllll}
\multicolumn{4}{c}{ for $\mathrm{O}-\mathrm{H}$ stretching band of water $\left(\mathrm{cm}^{-1}\right)$} \\
\hline PVP & & K15 & K30 & K90 \\
\hline Free water & 3618 & 3618 & 3616 & 3618 \\
Bound water of $-\mathrm{SO}_{3}{ }^{-}$ & 3550 & 3554 & 3551 & 3553 \\
Bulk water & 3446 & 3449 & 3449 & 3448 \\
Bound water of $\mathrm{Na}^{+}$ & 3292 & 3290 & 3292 & 3291 \\
\hline
\end{tabular}

$\mathrm{g} \cdot \mathrm{L}^{-1} \mathrm{PVP}-\mathrm{K} 15 、 \mathrm{~K} 30$ 和 $\mathrm{K} 90$ 后, 其峰高分别为 $0.64 、 0.59$ 和 0.45 .

根据子峰面积相对于总面积的方法求出每种 水的百分含量, 结果示于表 4. 由此可见, PVP 的加 人对表面活性剂长链间的自由水及 $\mathrm{Na}^{+}$结合水的 含量影响较小, 但使 $-\mathrm{SO}_{3}-$ 的结合水明显减少, 本 体水的含量明显增多. 说明 PVP 也存在于 $\mathrm{W} / \mathrm{O}$ 微 乳液滴内表面活性剂的极性基团附近. 但对结合水 和本体水影响正好与 $\mathrm{C}_{12} \mathrm{BE}$ 体系相反, 说明 $\mathrm{C}_{12} \mathrm{BE}$ 和 AOT 两种体系的差异. 由两种 $\mathrm{W} / \mathrm{O}$ 微乳液中 水分子的 $\mathrm{O}-\mathrm{H}$ 振动峰高度均随 PVP 分子量增大 而降低, 可以推测, 显微弱正电性的 PVP 与荷负电 的磺酸根发生缔合, 使磺酸根的结合水减少, 将一 部分结合水排挤到水核内部, 使本体水含量增大. 本体水含量增大有利于提高红外吸收峰强度, 然而, PVP/AOT 之间的缔合导致两者的极性基团的极 性减弱, 基团极性越弱, 红外吸收峰强度越弱 ${ }^{[19]}$, 或 许后者起主导作用, 因而观察到体系的红外吸收峰 强度降低。

\section{表 4 含不同 PVP 时 AOT / $n \mathrm{C}_{7} \mathrm{H}_{16} / \mathrm{H}_{2} \mathrm{O}$ W/O 微 乳液各种状态水的相对百分含量}

Table 4 Effect of PVP on the various water contents in $\mathrm{W} / \mathrm{O}$ microemulstion of AOT

\begin{tabular}{ccccc}
\hline PVP & & K15 & K30 & K90 \\
\hline Free water $(\%)$ & 5.50 & 5.30 & 5.80 & 4.90 \\
Bound water of $-\mathrm{SO}_{3}{ }^{-}(\%)$ & 23.0 & 18.2 & 17.8 & 17.9 \\
Bulk water $(\%)$ & 35.4 & 41.6 & 42.9 & 42.5 \\
Bound water of $\mathrm{Na}^{+}(\%)$ & 36.4 & 35.0 & 33.7 & 34.9 \\
\hline
\end{tabular}


为了探讨 $\mathrm{PVP}$ 对 $\mathrm{W} / \mathrm{O}$ 微乳液滴影响的机理, 利用稀释型乌氏粘度计测定不同浓度 PVP 溶液的 比浓粘度, 以比浓粘度对 PVP 浓度作图, 求得 PVP-K15、-K30 和 $-\mathrm{K} 90$ 的特性粘数 $[\eta]$ 分别为 $0.00076 、 0.00212$ 和 $0.014 \mathrm{~L} \cdot \mathrm{g}^{-1}$ (图略). 由此计 算出三种 PVP 在水溶液中的均方根末端距 $\left(r^{2}\right)^{1 / 2}$ 分别为 $6.6 、 14.5$ 和 $69.8 \mathrm{~nm}$. 根据 Kotlarchyk ${ }^{[21]}$ 给 出的反相微乳液水核半径的计算公式, 求得 $298 \mathrm{~K}$ 时该 $\mathrm{AOT} \mathrm{W} / \mathrm{O}$ 微乳液的水核半径 $R_{\mathrm{w}}=2.658$ $\mathrm{nm}$. 由此可知, 该 AOT W/O 微乳液的水核半径小 于 PVP 的均方根末端距. 鉴于此, 并考虑到 $\mathrm{Na}^{+}$的 结合水和表面活性剂长链间的自由水含量均未随 PVP 的加人而发生明显变化. 可以推测, PVP 大分 子既不是整体进人 AOT W/O 微乳液的内核. 也 没有进人表面活性剂烃基尾部区域。PVP 的存在 主要影响 $\mathrm{W} / \mathrm{O}$ 微乳液水核中本体水和结合水, 说 明 PVP 主要存在于 $\mathrm{W} / \mathrm{O}$ 微乳液水核与表面活性 剂极性基团之间的界面区域。

然而, PVP 对 $\mathrm{C}_{12} \mathrm{BE}$ 和 AOT 两种 $\mathrm{W} / \mathrm{O}$ 微乳 液水核中水的状态产生了不同影响. 对前者, PVP 的存在导致结合水的相对含量增大, 对后者却使结 合水相对含量减少. 可能的原因是: 在实验条件下, $\mathrm{C}_{12} \mathrm{BE}$ 分子以内盐形式存在, 结合水的能力较小, 而 且, 醇的存在导致部分 $\mathrm{C}_{12} \mathrm{BE}$ 分子通过其羧基与醇 差基间发生氢键缔合, 也减少了与水的结合。当体 系中加人 PVP 后, 带有微量正电荷的 PVP 分子可 与 $\mathrm{C}_{12} \mathrm{BE}$ 分子的某一荷负电部位缔合 ${ }^{[12]}$, 使 $\mathrm{C}_{12} \mathrm{BE}$ 分子显示出一定正电性, 从而导致其与水分子的结 合能力增强. 因而观察到结合水的相对含量增加. 因水核中的含水量一定, 则本体水必然减小. 对于 AOT W/O 微乳液, 不含 PVP 时, 水合作用较强的 $-\mathrm{SO}_{3}$ - 和 $\mathrm{Na}^{+}$离子会结合较大量的水, 而加人 PVP 后, 略带正电性的 PVP 分子与 $-\mathrm{SO}_{3}$ - 基团作用, 从 而削弱了 $-\mathrm{SO}_{3}$-与水的缔合. 原来与 $-\mathrm{SO}_{3}$ - 结合 的水被释放出来进人内核, 成为本体水, 因而观察 到本体水含量增加 .

\section{References}

1 Tapas T K De, Amarnath M. Adv. Colloid Interface Sci., 1995, 59: 95

2 Giuseppe O, Aldo S. J. Phys. Chem., 1993, 97:5430

3 Jain T K, Varshney V, Maitra A. J. Phys. Chem., 1989, 93: 7409

4 Kawai T, Shindo N, Kon-No K. Colloid Polym. Sci., 1995, 273: 195

5 Hauser H, Haering G, Pande A, et al. J. Phys. Chem., 1989, 93: 7869

6 Papoutsi D, Lianos P, Brown B. Langmuir, 1994, 10: 3402

7 Suarez MJ, Lang J. J. Phys. Chem., 1995, 99: 4626

8 Suarez MJ, Lery J, Lang J. J. Phys. Chem., 1993, 97: 9808

9 Lianos P, Modes S. Langmuir, 1992, 8: 105

10 Zajac J, Chorro C, Lindheimer M. Langmuir, 1997, 13: 1486

11 Florenzano F H, Dias L G. Langmuir, 1997, 13: 5756

12 Xu Guiying, Sui Weiping, Li Ganzuo. Acta Chimica Sinica, 1997, 55: 1179

[徐桂英, 隋卫平, 李干佐. 化学学报 (Huaxue Xuebao), 1997, 55: 1179]

13 Xu G Y, Yang Y L, Zhang L, et al. Materials Sci. Engineering C, 1999, 10: 47

14 Wu JX. Polyvinylpyrrolidone, in Water-Soluble Macromolecules, Ed. By Yan RX. Beijing: Chemistry Industry Press. 1998, 586p [ 吴稼祥, 聚乙烯吡略烷酮, 严瑞煊主编, 水溶性高分子, 北京: 化学工业出版社, 1998.586p]

15 Xu Guiying, Zhang Li, Yang Yanlian, et al. ed. Proceedings of the Fifth International Seminar on Surfactants and Detergents, Publishing Company of Shanxi Economy, Taiyuan, 1998, 252p

16 Li Q, Li WH, Weng SF, et al. Acta Phys. -Chim. Sin., 1997, 13: 438

[李 泉, 李维红, 翁诗甫, 物理化学学报( Wuli Huaxue Xuebao), 1997, 13: 438]

17 Li GZ, Guo R, Jin JZ, Xu GY, Formations and Theories on Microemultion, Beijing : Petroleum Industry Press. 1995, 48p [李干佐, 郭 荣, 金静芷, 徐桂英. 微乳液形成与理论, 北京: 石油工业出版社, 1995, 48p]

18 Painter P C, Snyder R W. Apple Spetrosco., 1981, 35: 475

19 Wu JG, Shi N, Gao HC, et al. Science in China Series B, 1983, 12: 107

[吴谨光, 施鼎, 高宏成等. 中国科学, B 辑( Zhongguo Kexue, B), 1983, 12: 107

20 Wolfgang M. Langmuir, 1996, 12: 1188

21 Kotlarchyk M. J. Phys. Chem., 1982, 86: 3273 


\title{
The State of Solubilized Water in W/O Microemulsions in the Presence of Polyvinylpyrrolidone*
}

\author{
Xu Gui-Ying Zhang Li Mao Hong-Zhi \\ ( Key Laboratory for Colloid \& Interface Chemistry of Education Ministry, \\ Shandong University, Jinan 250100) \\ Bao Meng Lu Yan \\ ( Department of Chemistry, Shandong University of Building Materials, Jinan 250022)
}

\begin{abstract}
Recently, high molecular weight additives such as proteins or synthetic polymer have been dissolved in water pools in $\mathrm{W} / \mathrm{O}$ microemulsions, which served as microreacter for enzymatic or chemical reactions. However, the report on the effect of different molecular weight polymers on the state of water in $\mathrm{W} / \mathrm{O}$ microemulsions made with zwitterionic surfactant has been so far scarcely investigated.

We report here the results of research concerning the effect of different molecular weight polyvinylpyrrolidones(PVP-K15, PVP-K30, PVP-K90, and PVP-K120) on the state of water in two kinds $\mathrm{W} / \mathrm{O}$ microemulsions made with water/dodecyl betaine $\left(\mathrm{C}_{12} \mathrm{BE}\right) / n$-pentanol $\left(\mathrm{C}_{5} \mathrm{OH}\right) / n$-heptane $\left(\mathrm{C}_{7} \mathrm{H}_{16}\right)$ (I), and water/ aerosol OT (AOT) $/ \mathrm{C}_{7} \mathrm{H}_{16}$ (II) using Fourier Transform Infrared(FTIR) spetroscopy.

FTIR spectra of all the samples were taken on a BIO-RED model 165 FTIR spectrometer using AgCl cells of a fixed path length of $0.025 \mathrm{~nm}$. All spectra were recorded at room temperature $\left(25{ }^{\circ} \mathrm{C}\right)$. Two kinds of $\mathrm{W} / \mathrm{O}$ microemulsions with PVP that the changes in the $\mathrm{O}-\mathrm{H}$ stretch peak of water molecule could be observed. The results indicate that the content of the bound water was increased, and the amount of the bulk water was decreased in the system (I) in the presence of the PVP. But the amount of bound water was decreased and the amount of the bulk water was increased in the system( II) as PVP was added. This is contributed to the interaction of PVP macromolecules with the head groups of amphiphile: the polarity of $\mathrm{C}_{12} \mathrm{BE}$ molecule was increased but the polarity of AOT molecule was decreased as PVP was added.
\end{abstract}

Keywords: FTIR, Polyvinylpyrrolidone(PVP), Dodecyl betaine $\left(\mathrm{C}_{12} \mathrm{BE}\right)$, Sodium bis(2-ethylhexylhexyl) sulfosuccinate(AOT), Water-in-oil microemulsion(W/O) 